\title{
Coexistence of long-range magnetic ordering and singlet ground state in the spin-ladder superconductor $\mathrm{SrCa}_{13} \mathrm{Cu}_{24} \mathrm{O}_{41}$
}

\author{
Guochu Deng, ${ }^{1, *}$ Michel Kenzelmann, ${ }^{2}$ Sergey Danilkin, ${ }^{1}$ Andrew J. Studer, ${ }^{1}$ Vladimir Pomjakushin, ${ }^{3}$ Paolo Imperia, ${ }^{1}$ \\ Ekaterina Pomjakushina, ${ }^{2}$ and Kazimierz Conder ${ }^{2}$ \\ ${ }^{1}$ Bragg Institute, Australian Nuclear Science and Technology Organisation, Locked Bag 2001, Kirrawee DC, NSW 2232, Australia \\ ${ }^{2}$ Laboratory for Developments and Methods, Paul Scherrer Institute, CH-5232 Villigen, Switzerland \\ ${ }^{3}$ Laboratory for Neutron Scattering, Paul Scherrer Institute, CH-5232 Villigen, Switzerland
}

(Received 23 August 2013; published 26 November 2013)

\begin{abstract}
A long-range magnetic order was discovered in the quasi-one-dimensional spin-ladder compound $\mathrm{SrCa}_{13} \mathrm{Cu}_{24} \mathrm{O}_{41}$ by susceptibility, specific heat, and neutron diffraction experiments. The temperature dependence of the magnetic Bragg peak intensity could be well fitted to the power law with a transition temperature $T_{N}=$ $4.23 \mathrm{~K}$ and a critical exponent $\beta=0.28$, indicating a three-dimensional phase transition for a low-dimensional magnet. A computer program was coded and found two possible magnetic structure models fitting best with all the observed magnetic peaks. These models suggest the spin-ladder sublattice is magnetically ordered with $\mathrm{Cu}$ moments aligning along the $a$ axis. The spin interactions are primarily antiferromagnetic along rungs and legs, while there are ferromagnetic clusters along legs. Surprisingly, the singlet-triplet spin-gap excitation is observable above and below $T_{N}$, indicating a coexistence of the spin-singlet ground state and long-range magnetic ordering state in this compound.
\end{abstract}

DOI: 10.1103/PhysRevB.88.174424

PACS number(s): 75.10.Kt, 75.30.-m, 75.30.Hx, 75.40.-s

\section{INTRODUCTION}

In the last two decades, many predictions for onedimensional (1D) magnets, such as Haldane's conjecture on spin chains ${ }^{1}$ and Dagotto's model on spin ladders, ${ }^{2,3}$ have been proved experimentally., ${ }^{4,5}$ This progress has strongly excited researchers' interests in low-dimensional magnetic systems and might help us understand more complex materials such as high temperature superconductors. Dagotto et al. ${ }^{2}$ and Rice et $a l .{ }^{3}$ also predicted that spin ladders with even legs can become either superconducting or develop a charge density wave when doped with an appropriate level of holes. Experimentally, it was discovered that the two-leg spin-ladder compounds $\mathrm{Sr}_{14-x} \mathrm{Ca}_{x} \mathrm{Cu}_{24} \mathrm{O}_{41}$ ( $\mathrm{SrCa} x$ with $x>10$ ) become superconducting under pressure, ${ }^{6}$ drawing extensive attention to Dagotto's prediction and this series. ${ }^{7}$ In spite of numerous efforts, it is still widely debated whether superconductivity in $\mathrm{SrCa} x$ originates from the hole-pairing mechanism suggested by Dagotto et al. ${ }^{2}$ As the only known spin-ladder superconductor, it is thus of high importance to understand its electronic and magnetic properties, especially the ground state at low temperature and high pressure.

The series SrCax consist of edge-shared $\mathrm{CuO}_{2}$ chains and two-leg $\mathrm{Cu}_{2} \mathrm{O}_{3}$ ladders, which are stacked along the $b$ axis and run incommensurately along the $c$ axis. SrCax compounds are inherently hole doped with an average $\mathrm{Cu}$ valence of around +2.25 . In the parent compound $\mathrm{Sr}_{14} \mathrm{Cu}_{24} \mathrm{O}_{41}$ ( $\left.\mathrm{SrCa} 0\right)$, most of the doped holes were believed to be located on chain sites, forming a Zhang-Rice singlet $\left(\mathrm{Cu}^{3+}\right)$ at low temperature. ${ }^{8}$ Bridged by a Zhang-Rice singlet, two neighbor $\mathrm{Cu}^{2+}$ form an antiferromagnetic (AFM) dimer $\left(\mathrm{Cu}^{2+}(\uparrow)-\mathrm{Cu}^{3+}-\mathrm{Cu}^{2+}(\downarrow)\right)$ on chains. The spin ladders in $\mathrm{SrCa} x$ have a similar structure to the two-leg ones in $\mathrm{SrCu}_{2} \mathrm{O}_{3}$ and feature a gapped-singlet ground state below room temperature. ${ }^{5}$ A particularly fascinating behavior has been observed in the Ca-doped SrCax samples. ${ }^{7}$ Even though $\mathrm{Ca}$ substitution for $\mathrm{Sr}$ is isovalent, holes transfer from chains to ladders with increasing $\mathrm{Ca}$ doping, causing substantial changes in the physics of both ladders and chains. ${ }^{9}$ One surprise is the discovery of a superconducting phase under hydrostatic pressure in $\operatorname{SrCa} x$ with $x \geqslant 10$. However, the ground state for both chains and ladders in the SrCax compounds with $x \geqslant 10$ at ambient pressure is still under active debate. For example, K. Kumagai et al. ${ }^{10}$ speculated that the spin gap of the ladder collapses at $x \sim 13$ with the nuclear magnetic resonance (NMR) measurements. However, we recently observed the spin-gap excitation in the compound with $x=13 .^{11}$ Another noteworthy fact is that long-range magnetic ordering was suggested from the susceptibility measurement in the powder samples, ${ }^{12}$ which was confirmed by T. Nagata et al. ${ }^{13}$ with neutron scattering. The coexistence of the long-range magnetic ordering and spin-gapped states was observed in various $1 \mathrm{D}$ magnetic systems, e.g., Si-doped $\mathrm{CuGeO}_{3}$ (Ref. 14) and $\mathrm{CsNiCl}_{3}$ (Ref. 15). The corresponding theoretical work demonstrated that these two states in each system originate from the same chemical lattice in a specific temperature range. ${ }^{16,17}$ However, the origin of the magnetic ordering and spin-gapped states in $\mathrm{SrCa} x$ is still not clear.

In this paper, we systematically investigated the static and dynamic behaviors of the newly grown SrCa13 single crystal in order to characterize the ground state in this compound. We measured the magnetic susceptibility and heat capacity and observed an anomaly at $\sim 4 \mathrm{~K}$, providing evidence for a magnetic phase transition. Magnetic order was directly observed through the measurement of magnetic Bragg peaks in $a c$ and $b c$ planes using neutron diffraction. Considering a wide range of magnetic structure models, we found two magnetic models that give a good agreement with the observed magnetic intensities. The $\mathrm{Cu}^{2+}$ moments on the ladder sublattice are aligned along the $a$ axis. The antiferromagnetic coupling overwhelms the exchanges through rungs and legs, while some local ferromagnetic (FM) clusters exist in the magnetic phase. On the other hand, the singlet-triplet spin-gap excitation was found to survive below $T_{N}$ by inelastic neutron scattering. We 
argue that there is a scenario of coexistence of long-range magnetic ordering and singlet ground states in the spin-ladder compound SrCa13.

\section{EXPERIMENTAL}

A $\mathrm{SrCa} 13$ single crystal was grown by using a traveling solvent-floating zone technique with 35 Bar high oxygen pressure. ${ }^{18}$ High resolution $\mathrm{x}$-ray powder diffraction experiments with the powderized samples from different parts of the crystal boule were carried out on a Bruker D8 $\mathrm{x}$-ray diffractometer, confirming the phase purity of the as-grown single crystal. ${ }^{18}$ The magnetic susceptibility was measured using a superconducting quantum interference device (SQUID) magnetometer (Cryogenic Ltd.). The neutron scattering experiments were done on the thermal neutron triple-axis spectrometer TAIPAN and the high flux neutron powder diffractometer WOMBAT, both located at the high flux beam reactor OPAL in Australian Nuclear Science and Technology Organization (ANSTO). A fixed final energy mode $\left(E_{\mathrm{f}}=14.87 \mathrm{meV}\right)$ with vertically focused monochromator was used for the experiments on TAIPAN. The sample for the neutron experiment was cylindrical with a diameter of $6 \mathrm{~mm}$ and a height of $15 \mathrm{~mm}$. The experiments on WOMBAT were done by using an incident beam with $\lambda=2.41 \AA$. All the reflections used in this paper are based on the average unit cell $(a=11.26, b=12.46, c=27.46)$ of SrCa13 with $c=7 c_{\text {ladder }} \approx 10 c_{\text {chain. }}$. The magnetic structure fittings were done by Fullprof, ${ }^{19}$ which was invoked by a computer program written in $\mathrm{C}++$. The $\mathrm{C}++$ program is responsible for creating different magnetic models and testing the goodness of the fittings.

\section{RESULTS AND DISCUSSIONS}

Figure 1(a) shows the magnetic susceptibility from the single crystal sample of SrCa13 along the $b$ and $c$ axes. Because of the large spin-ladder gap, the ladder contribution to the susceptibility is small at low temperature. As a result, the magnetic susceptibility arises mostly from the spin chains at low temperature. According to the spin-dimer model used in Carter et al.'s work, ${ }^{20}$ the chain contribution to the susceptibility is given by:

$$
\chi_{\text {chain }}=2 N_{D} g^{2} \mu_{B}^{2} /\left[k_{B} T\left[3+\exp \left(J_{D} / k_{B} T\right)\right]\right],
$$

where $N_{D}$ is the number of dimer per formula unit (f.u.), $\mu_{B}$ is the Bohr magneton, $k_{B}$ is the Boltzmann's constant, and $J_{D}$ is the intradimer exchange energy. Due to the hole doping, unpaired $\mathrm{Cu}$ spins exist on both chains and ladders, leading to a Curie tail at low temperature whose susceptibility is given by $\chi_{\mathrm{CW}}=N_{F} g^{2} \mu_{B}^{2} /\left[4 k_{B} T(T-\theta)\right]$, where $N_{F}$ is number of free spins per f.u. The experimentally observed magnetic susceptibility [see the inset in Fig. 1(a)] could be well fitted to a sum of these two contributions. For the magnetic susceptibility along the $b$ axis, we obtained $J_{D}=136.2 \mathrm{~K}$, $N_{D}=1.05$, and $N_{F}=1.64$. The observed $J_{D}$ is very close to the previous reported value $133 \mathrm{~K}$ in $\mathrm{SrCa} 0$. However, the dimer number $N_{D}=1.05$ is somewhat less than the reported 1.47 in $\mathrm{SrCa} 8$, while the free spin number $N_{F}=1.67$ is much higher than that value (0.55) in $\mathrm{SrCa} 8$ (Ref. 20). This is most likely the result of an increased hole transfer to ladders for the higher Ca doping in SrCa13.

In order to do further analysis, the original susceptibility data were subtracted with the fitted Curie tail below $T=5 \mathrm{~K}$ and plotted in Fig. 1(a). The resulting magnetic susceptibility reveals a broad maximum around $80 \mathrm{~K}$, which corresponds to the contribution of the $\mathrm{Cu}$ spin dimers on the chain sublattice. ${ }^{20}$ Additionally, a sharp peak, indicated by a black arrow, was observed at $\sim 4.2 \mathrm{~K}$ in the susceptibility data, indicating that it corresponds to the onset of antiferromagnetic order. In spite of its weakness, this anomaly is also visible in the raw data without the Curie tail subtraction.

Specific heat $C_{p}$ was measured for the $\mathrm{SrCa} 13$ single crystal sample to further clarify the origin of the anomaly. $C_{p}-T$ and $C_{p} / T-T$ were plotted in Fig. 1(b) and its inset, respectively.
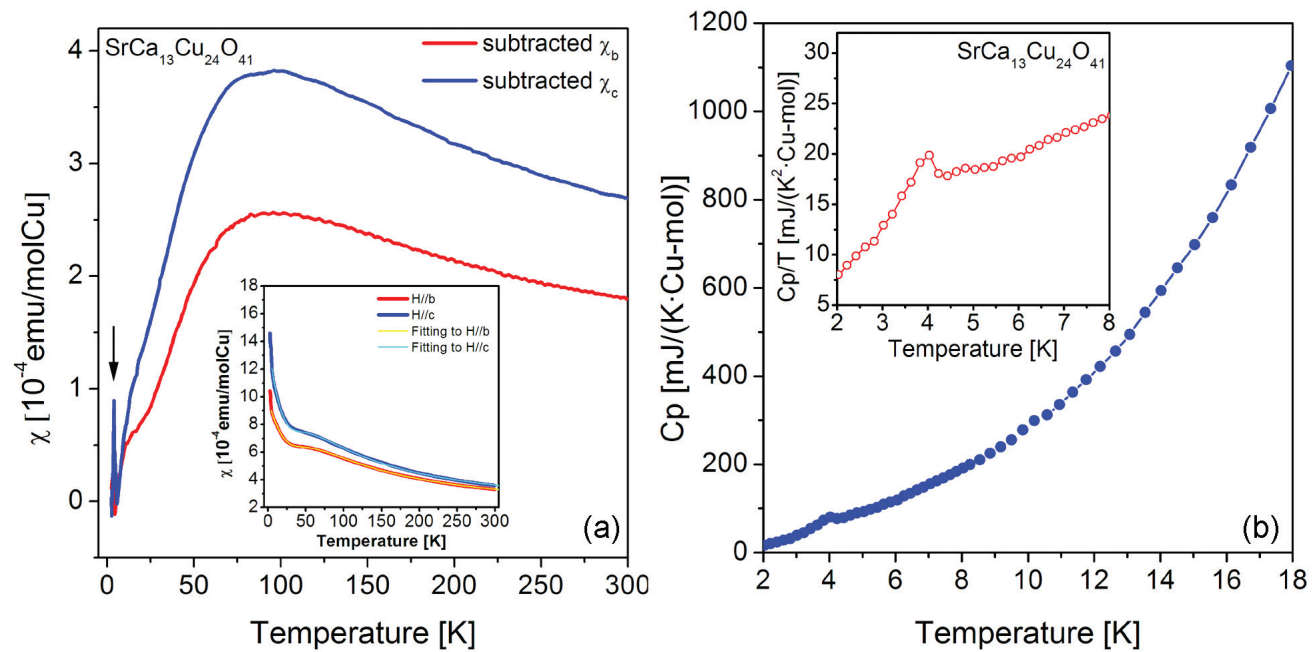

FIG. 1. (Color online) (a) Temperature dependence of magnetic susceptibility of a SrCa13 single crystal along $b$ and $c$ axes in which the Curie-Weiss term was subtracted, as described in the text. The black arrow points to the sharp peak at $\sim 4 \mathrm{~K}$ after subtraction. The inset shows the original susceptibility data without any subtraction. (b) Temperature dependency of specific heat of a SrCa13 single crystal sample at a zero magnetic field. The inset shows the $C_{p} / T$ versus temperature curve. 

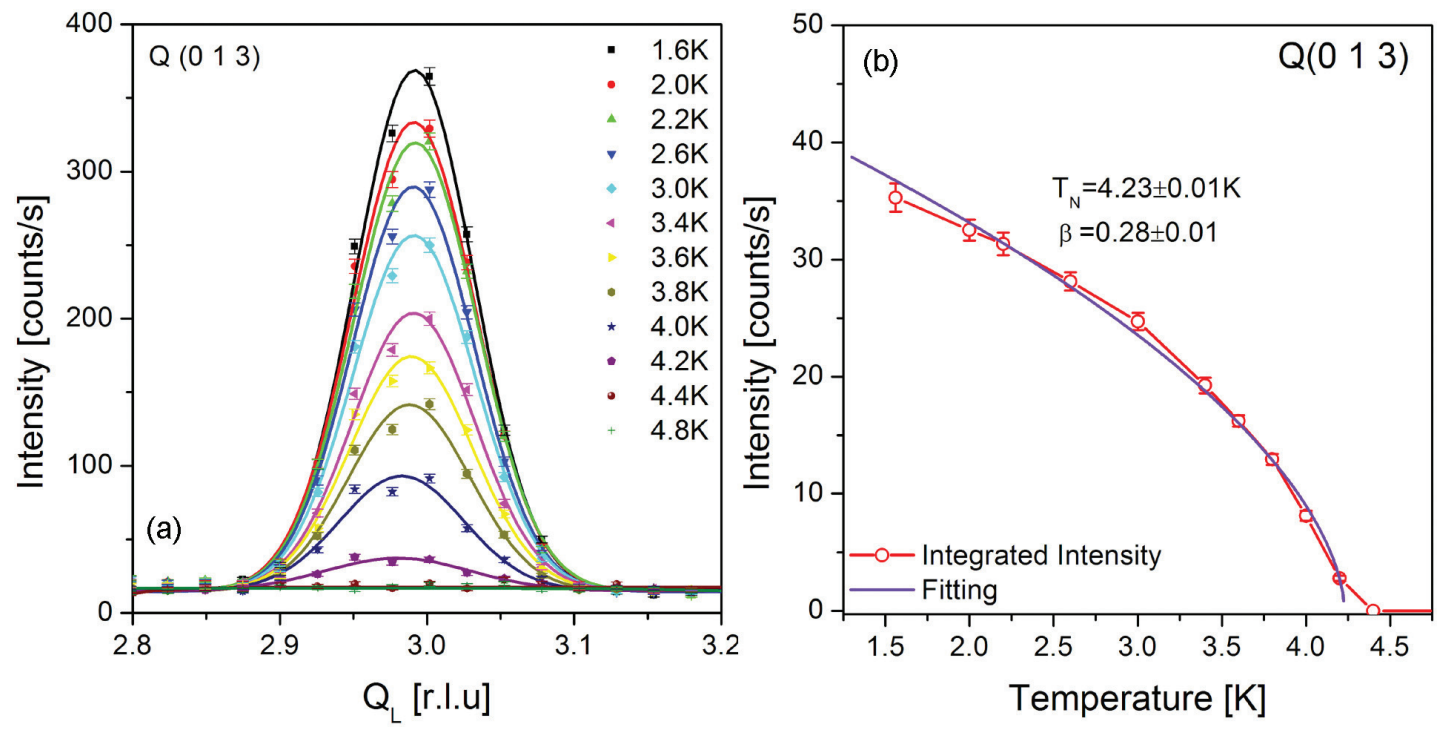

FIG. 2. (Color online) (a) Magnetic Bragg peak (013) at different temperatures near Néel temperature. (b) Temperature dependency of the intensity of Bragg peak (013); the red circles show the peak intensity, and the purple curve is fitted to it.

A weak anomaly is clearly visible near $4.2 \mathrm{~K}$ and is thus consistent with the anomaly in the susceptibility. The anomaly becomes even clearer in the $C_{p} / T-T$ plot. Such a $\lambda$-type anomaly demonstrates that this is a transition to a long-range magnetic order rather than spin-glass freezing. ${ }^{21}$

In order to elucidate the origin of the observed phase transition above, we performed neutron diffraction experiments for a SrCa13 single crystal sample. The temperature dependence of the intensity of Bragg peaks (013) and (107) were measured on the three-axis neutron spectrometer TAIPAN. The temperature dependence of the peak intensity at $Q(013)$ is shown in Fig. 2(a). It gradually decreases with increasing temperature from $1.5 \mathrm{~K}$ to $4.2 \mathrm{~K}$ and disappears above $4.2 \mathrm{~K}$. This indicates a second-order nature of this phase transition in SrCa13. A similar temperature dependence was also measured at (107) Bragg peak, which disappeared at the same temperature that (013) did. The agreement of the transition temperature from the neutron diffraction and specific heat measurements confirms that all of these observed phenomena originated from the same magnetic transition.

The integrated intensity vs temperature curve for (013) is shown in Fig. 2(b). It could be fitted to a simple power law: $I=A\left(T_{N}-T\right)^{2 \beta}$, where $I$ is intensity, $A$ is a scaling factor, $T_{N}$ is the Néel temperature, and $\beta$ is the critical exponential. The fit yields for $T_{N}=4.23 \pm 0.01 \mathrm{~K}$ and $\beta=0.28 \pm$ 0.01 . The fitted $T_{N}$ value is close to the $4.2 \mathrm{~K}$ obtained from the specific heat measurement, and the difference probably arises from a slightly different calibration of the thermometers. The exponent $\beta$ is larger than the exponent for the twodimensional (2D) Ising model but smaller than the one for the three-dimensional (3D) Heisenberg model, which is $\beta=$ 0.367 . Similar exponents have also been observed in other 1D magnets featuring 3D magnetic order, e.g., $\mathrm{BaCu}_{2} \mathrm{Si}_{2} \mathrm{O}_{7}(\beta=$ 0.25 ; Ref. 22) and $\mathrm{CsNiCl}_{3}(\beta=0.27$; Ref. 23).

Our experimental results clearly show that $\mathrm{SrCa} 13$ undergoes a long-range magnetic transition around $4 \mathrm{~K}$. We now attempt to characterize the structure of the magnetic phase. Since the system has an incommensurate composite crystal structure, ${ }^{9}$ describing the chemical structure is quite complicated. As we will see, this also complicates the description of the symmetry of the magnetic structure. In a simplification, the nuclear structure can be described in an average unit cell with $c=7 c_{\text {ladder }} \approx 10 c_{\text {chain }}$. In such a large unit cell, there are seven $\mathrm{Cu}$ spins in each leg of one spin ladder and $10 \mathrm{Cu}$ spins in each chain. The whole unit cell has four spin ladders and four spin chains (in two layers), for a total of $96 \mathrm{Cu}$ spins.

We measured the magnetic peaks of the SrCa13 single crystal sample by using the diffractometer WOMBAT below and above $T_{N}$. Two crystal planes (namely, $a c$ and $b c$ planes) were aligned in the horizontal scattering plane and mapped by vertically rotating the crystal sample. The diffraction patterns were combined and converted into a $2 \mathrm{D}$ mapping of the corresponding reciprocal planes covering at least one quadrant. Figures 3(a) and (b) show some parts of the mapped $(0 \mathrm{kl})$ and $(h 0 l)$ reciprocal planes at $1.5 \mathrm{~K}$ and $10 \mathrm{~K}$, respectively.

Figure 3(a) shows that the two different diffraction peaks at $1.5 \mathrm{~K}$ disappeared on heating to $10 \mathrm{~K}$. They correspond to a wave-vector transfer $\mathbf{Q}=(107)$ and (507). In Fig. 3(a), we show five magnetic peaks that appear when cooling down to $1.5 \mathrm{~K}$. They are (013), (033), (053), (073), and (093). By subtracting of the mapping results at $1.5 \mathrm{~K}$ and $10 \mathrm{~K}$, it is possible to identify more magnetic peaks in both $(0 \mathrm{kl})$ and $(h 0 l)$ planes. A schematic diagram of the observed magnetic Bragg peak in the $b c$ plane is shown in Fig. 4. The integrated intensities of the magnetic peaks were corrected with Lorentz factor and listed in Table I. It is worthwhile to mention that Nagata et al..$^{24}$ only observed six magnetic reflections in the SrCa11.5 single crystal sample by neutron diffraction. Table I also shows a few Bragg peaks sitting where no magnetic intensity was observed in our experiment, which provides more constraints on the magnetic structure refinement.

The refinement of the magnetic structure represents a challenge, first because of the large unit cell that contains 

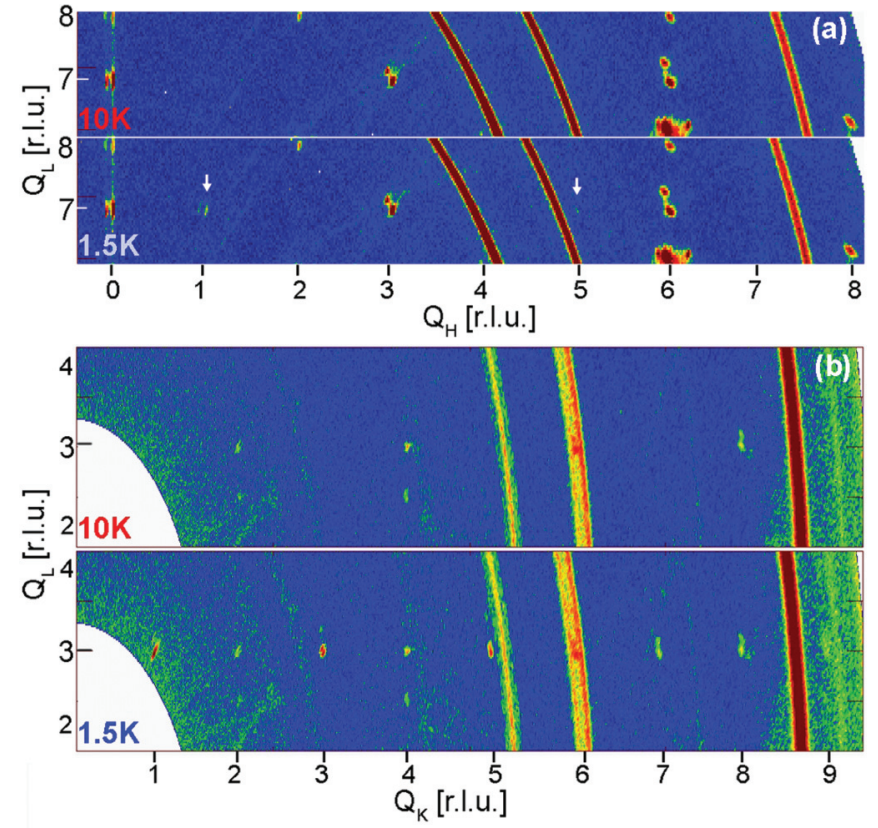

FIG. 3. (Color online) Some magnetic peaks observed by neutron diffraction in the $a c$ (a) and $b c$ (b) reciprocal planes of $\mathrm{SrCa} 13$ at $1.5 \mathrm{~K}$ and $10 \mathrm{~K}$. The white arrows in (a) point out the emerged Bragg peak at low temperature. The ring-shape stripes are attributed to the $\mathrm{Al}$ sample holder in the cryostat.

96 magnetic ions and second because the number of degrees of freedom cannot be easily reduced due to the chemical complexity of the material. We note that the magnetic peaks cannot be indexed with an ordering wave vector of either the chain or ladder chemical lattice. This means that the magnetic order may be described by an ordering wave vector of the superspace group. To simplify the analysis, the magnetic structure models were built on the basis of the approximated average structure model, which was generated from the

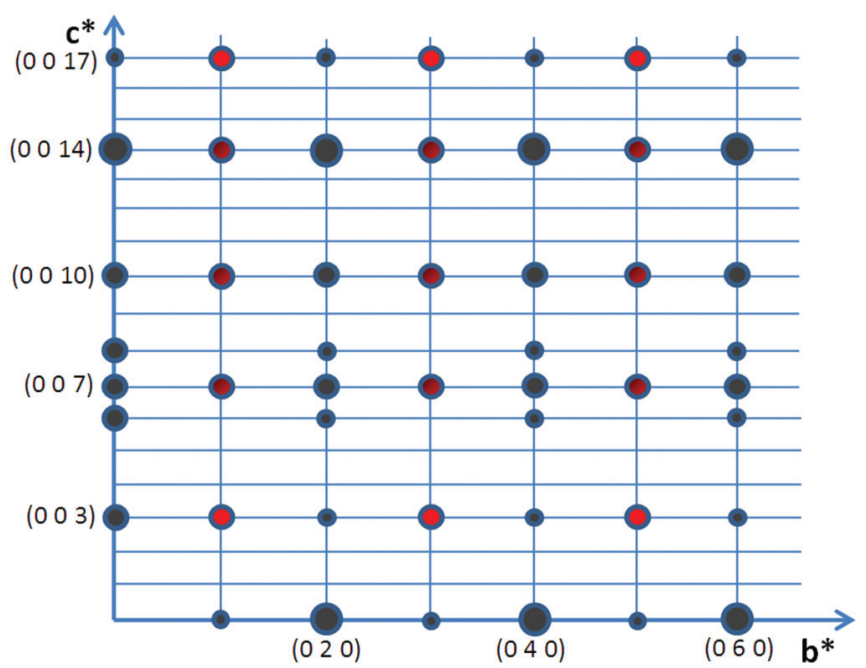

FIG. 4. (Color online) Schematic diagram of the $(0 k l)$ plane in the first quadrant of reciprocal space of $\mathrm{SrCa} 13$. The dark gray circles denote the nuclear peaks, while the red circles denote magnetic peaks. The dark red circles show the overlapping of the nuclear and magnetic peaks.
TABLE I. Observed integrated intensities $\left(I_{\mathrm{obs}}\right)$ and model A calculated intensities $\left(I_{\text {calc }}\right)$ of magnetic Bragg reflections in $\mathrm{SrCa}_{13} \mathrm{Cu}_{24} \mathrm{O}_{41} . I_{\text {obs }}$ values were measured at $T=1.5 \mathrm{~K}$. The reflections with $*$ means no apparent intensities were observed in the measurement, thus they were set to zero for refinement.

\begin{tabular}{|c|c|c|c|}
\hline$(h k l)$ & $I_{\mathrm{obs}}$ & Err & $I_{\text {calc }}$ \\
\hline$\left(\begin{array}{lll}0 & 0 & 3\end{array}\right)$ & 0 & - & 0 \\
\hline$\left(\begin{array}{lll}0 & 1 & 3\end{array}\right)$ & 134.02 & 3.32 & 142.7137 \\
\hline$\left(\begin{array}{lll}0 & 2 & 1\end{array}\right)$ & 0 & - & 0.0234 \\
\hline$\left(\begin{array}{lll}0 & 2 & 3\end{array}\right)$ & 0 & - & 0.0286 \\
\hline$\left(\begin{array}{lll}2 & 0 & 5\end{array}\right)$ & 0 & - & 2.7916 \\
\hline$\left(\begin{array}{lll}0 & 3 & 3\end{array}\right)$ & 117.49 & 2.48 & 136.4064 \\
\hline$\left(\begin{array}{lll}0 & 1 & 7\end{array}\right)$ & 54.4 & 7.02 & 60.6219 \\
\hline$\left(\begin{array}{lll}1 & 0 & 7\end{array}\right)$ & 55.57 & 1.99 & 33.8687 \\
\hline$\left(\begin{array}{lll}2 & 0 & 7\end{array}\right)$ & 0 & - & 5.3063 \\
\hline$\left(\begin{array}{lll}0 & 4 & 1\end{array}\right)$ & 0 & - & 0.0693 \\
\hline$\left(\begin{array}{lll}0 & 4 & 3\end{array}\right)$ & 0 & - & 0.0828 \\
\hline$\left(\begin{array}{lll}0 & 3 & 7\end{array}\right)$ & 47.72 & 13.4 & 58.1333 \\
\hline$\left(\begin{array}{lll}0 & 5 & 2\end{array}\right)$ & 0 & - & 8.3249 \\
\hline$\left(\begin{array}{lll}0 & 1 & 10\end{array}\right)$ & 53.12 & 8.89 & 79.9475 \\
\hline$(047)$ & 0 & - & 0.7599 \\
\hline$\left(\begin{array}{lll}0 & 5 & 3\end{array}\right)$ & 95.21 & 4.4 & 108.0833 \\
\hline$\left(\begin{array}{lll}4 & 0 & 7\end{array}\right)$ & 0 & - & 1.1076 \\
\hline$\left(\begin{array}{lll}0 & 6 & 1\end{array}\right)$ & 0 & - & 0.0975 \\
\hline$\left(\begin{array}{lll}0 & 6 & 3\end{array}\right)$ & 0 & - & 0.1164 \\
\hline$\left(\begin{array}{lll}0 & 3 & 10\end{array}\right)$ & 59.25 & 10.01 & 58.5897 \\
\hline$(057)$ & 46.75 & 10.52 & 49.0892 \\
\hline$\left(\begin{array}{lll}5 & 0 & 7\end{array}\right)$ & 17.07 & 4.09 & 1.4647 \\
\hline$\left(\begin{array}{lll}0 & 1 & 14\end{array}\right)$ & 9.24 & 11.29 & 29.4831 \\
\hline$\left(\begin{array}{lll}0 & 6 & 10\end{array}\right)$ & 23.59 & 6.65 & 36.1175 \\
\hline$\left(\begin{array}{lll}0 & 3 & 14\end{array}\right)$ & 33.36 & 14.87 & 26.1004 \\
\hline (0 7 3) & 84.33 & 5.21 & 73.0731 \\
\hline$\left(\begin{array}{lll}0 & 7 & 1\end{array}\right)$ & 0 & - & 8.2398 \\
\hline (0 7 2 2) & 0 & - & 3.1587 \\
\hline (0 7 7) & 54.29 & 8.34 & 36.5239 \\
\hline$\left(\begin{array}{lll}0 & 1 & 17\end{array}\right)$ & 33.44 & 3.61 & 36.3669 \\
\hline 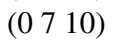 & 9.31 & 7.19 & 19.1552 \\
\hline 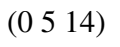 & 21.26 & 14.61 & 19.8942 \\
\hline$\left(\begin{array}{lll}0 & 3 & 17\end{array}\right)$ & 52.56 & 4.28 & 36.4887 \\
\hline$\left(\begin{array}{lll}6 & 0 & 7\end{array}\right)$ & 0 & - & 0.1116 \\
\hline$\left(\begin{array}{lll}0 & 8 & 3\end{array}\right)$ & 0 & - & 0.1132 \\
\hline$\left(\begin{array}{lll}0 & 9 & 1\end{array}\right)$ & 0 & - & 5.6404 \\
\hline
\end{tabular}

incommensurate modulation model from our previous research work. ${ }^{9}$ In order to simplify the magnetic structure model, we assumed only collinear magnetic structures, and we tested the chain and ladder magnetic structure models separately by assuming only ladders or chains order. For each sublattice, the magnetic moment orientation was tested for the three main axes. A series of $\mathrm{C}++$ programs was coded to build and test various magnetic structure models by invoking Fullprof for the refinement. We found that only chain-sublattice ordering cannot explain the observed magnetic Bragg peaks. The ladder spin sublattice must be ordered. Two best solutions were found for the ordered ladder sublattice by using this sifting method with a criteria of the fitting parameters like $R_{F}^{2}$ and $\chi^{2}$ etc. The spin configurations of these two models are exhibited in Fig. 5.

The $F_{\text {obs }}-F_{\text {calc }}$ plot in Fig. 6 shows how close the observed and calculated intensities are. The fitting parameters for each 


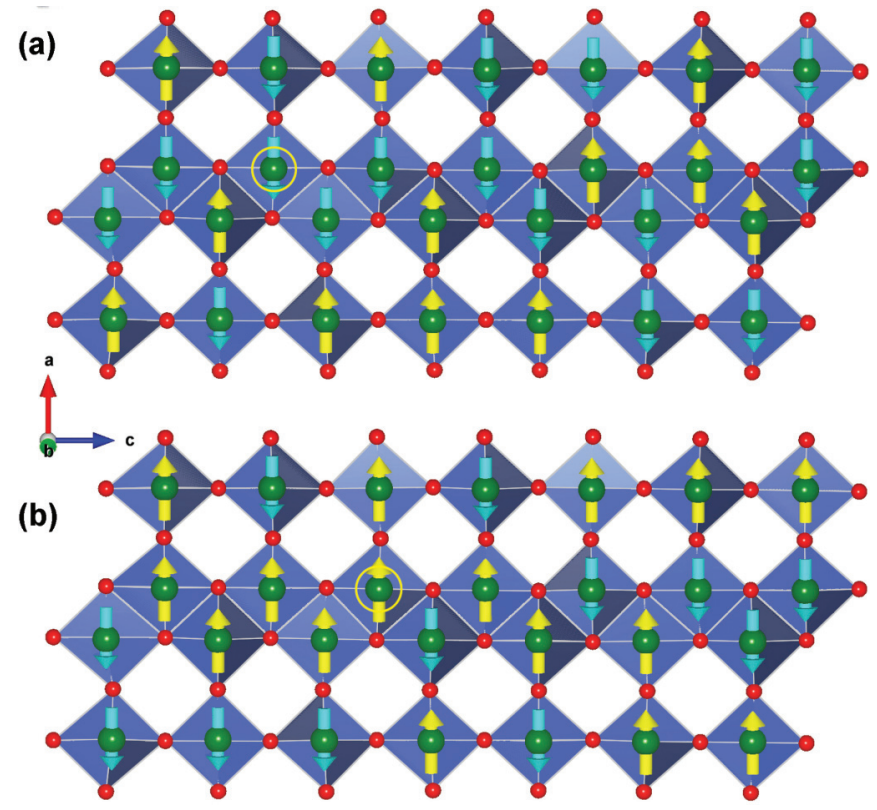

FIG. 5. (Color online) Magnetic structure models A and B for the ordered ladder sublattice. Only the first layers are shown here; the second layer has the same configuration but with flipping all spins and exchanging the two ladder positions. The $\mathrm{Cu}$ position marked with a yellow circle is possibly taken by a hole in each model.

model are listed in the plot. The calculated intensities of both magnetic structure models match the measured intensities very well. Considering that the other parameters, such as the approximate nuclear structure, Debye-Waller factors, etc., are fixed with the exception of the spin configuration, the agreement of these two models with the data is reasonably good. The remaining difference between the model and the data could arise from the use of an average structural model instead of the incommensurate model, the omission of the existence of holes on the ladder lattice, or a canting of the magnetic moments.

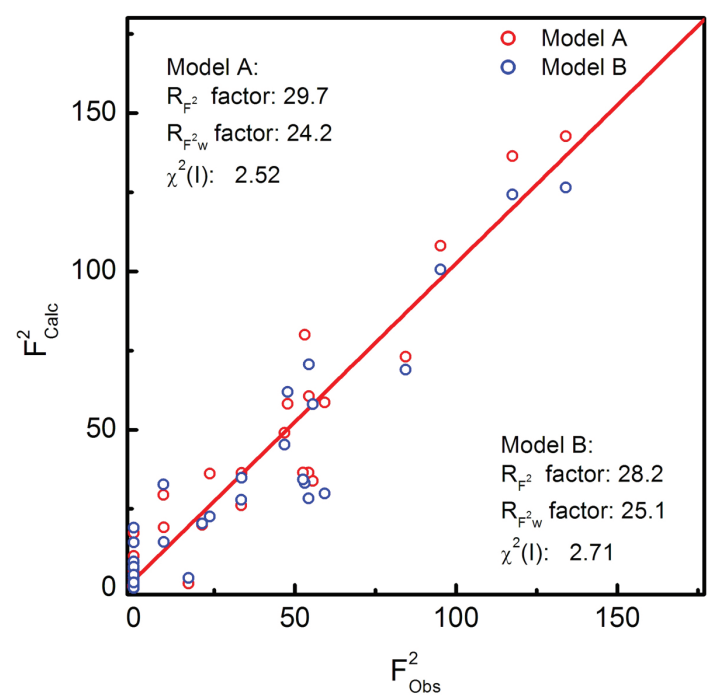

FIG. 6. (Color online) The observed intensities of the magnetic Bragg reflections at $1.5 \mathrm{~K}$ are plotted against the values calculated from the magnetic structure model $\mathrm{A}$ and $\mathrm{B}$.
In addition, the spin-chain sublattice could be ordered at the same time. However, when adjusting the $\mathrm{Cu}$-chain moments, the refinement yielded only relatively small $\mathrm{Cu}$ moments compared to those on ladders. This is in contrast to NMR data that indicate that the $\mathrm{Cu}$-chain moments are significantly larger than the $\mathrm{Cu}$-ladder moments. ${ }^{25}$ It is possible that the $\mathrm{Cu}$-chain spin are only partially ordered. The magnetic moment is averaged when all the $\mathrm{Cu}$ spins on chains are taken into account. Since the number of fitting parameters is increased in this process, it is also possible that the $\mathrm{Cu}$-chain moments remain disordered. However, we have tested whether only chain ordering without ladder ordering could explain the diffraction data, and we can exclude such a possibility. It is worth mentioning that the uncertainty of the state of the $\mathrm{Cu}$ moments on the chain sublattice also causes the difficulty in determining the size of $\mathrm{Cu}$ moments on the ladder sublattice.

The two models in Fig. 5 share several interesting common features. First of all, the nearest next-neighbor spins of both models mostly have an AFM interaction along rungs and legs. Some ferromagnetic clusters $(3 \sim 4 \mathrm{Cu}$ atoms $)$ can be found along the legs in both models. By measuring six magnetic peaks, T. Nagata et al. ${ }^{24}$ had proposed a magnetic structural model for SrCa11.5. Their model, in which both the chain and ladder sublattices are magnetically ordered, is based on an ad-hoc assumption that the magnetic moments on both chains and ladders are along the $a$ axis. ${ }^{24}$ Nagata's model showed that most $\mathrm{Cu}-\mathrm{Cu}$ spin interactions along rungs and legs are ferromagnetic while they are mainly antiferromagnetic in our models. Considering the nearly $180^{\circ} \mathrm{Cu}-\mathrm{O}-\mathrm{Cu}$ bond angle along rungs/legs, an antiferromagnetic coupling through rungs/legs is more reasonable. The larger number of observed reflections in our experiments makes the structure model proposed in this work more reliable.

The most striking fact about our magnetic models is its compatibility with hole doping. As shown in Fig. 5, inserting a hole near ferromagnetic clusters (marked with the yellow circle in each model), the fitting results do not substantially change. However, when substituting other $\mathrm{Cu}^{2+}$ away from ferromagnetic clusters, the fitting gets tremendously worse. This hints that the local ferromagnetic clusters could be induced by the doped holes.

Long-range magnetic ordering state was reported in a lower Ca-doped composition ( $\mathrm{SrCa} 11.5)$ of the same spin-ladder series by Nagata et $a l .{ }^{24}$ In another two-leg spin-ladder compound, namely $\mathrm{SrCu}_{2} \mathrm{O}_{3}$ (Ref. 21), which features the same ladder structure as in $\mathrm{Sr}_{14-x} \mathrm{Ca}_{x} \mathrm{Cu}_{24} \mathrm{O}_{41}$, it was found that long-range magnetic ordering state can be induced by doping with nonmagnetic impurity $\mathrm{Zn}$ on $\mathrm{Cu}$ sites. Furthermore, the Néel temperature of this magnetic phase increases with the doping level of $\mathrm{Zn} .^{21} \mathrm{~A}$ very similar $T_{N}$ dependence on the Ca-doping level was reported in the powder sample in $\mathrm{Sr}_{14-x} \mathrm{Ca}_{x} \mathrm{Cu}_{24} \mathrm{O}_{41}$ by Isobe et al. ${ }^{12}$ The similarities of the crystal structures and doping effects in these two spin-ladder compounds hint that the long-range magnetic ordering could be also induced by impurities in $\mathrm{Sr}_{14-x} \mathrm{Ca}_{x} \mathrm{Cu}_{24} \mathrm{O}_{41}$, just like $\mathrm{SrCu}_{2} \mathrm{O}_{3}$.

It is well known that nonmagnetic impurities have various impacts on the behavior of low-dimensional magnetic systems. A particularly striking example is the observation of fractional spin $\frac{1}{2}$ edge state in the Haldane gap integer spin compound 

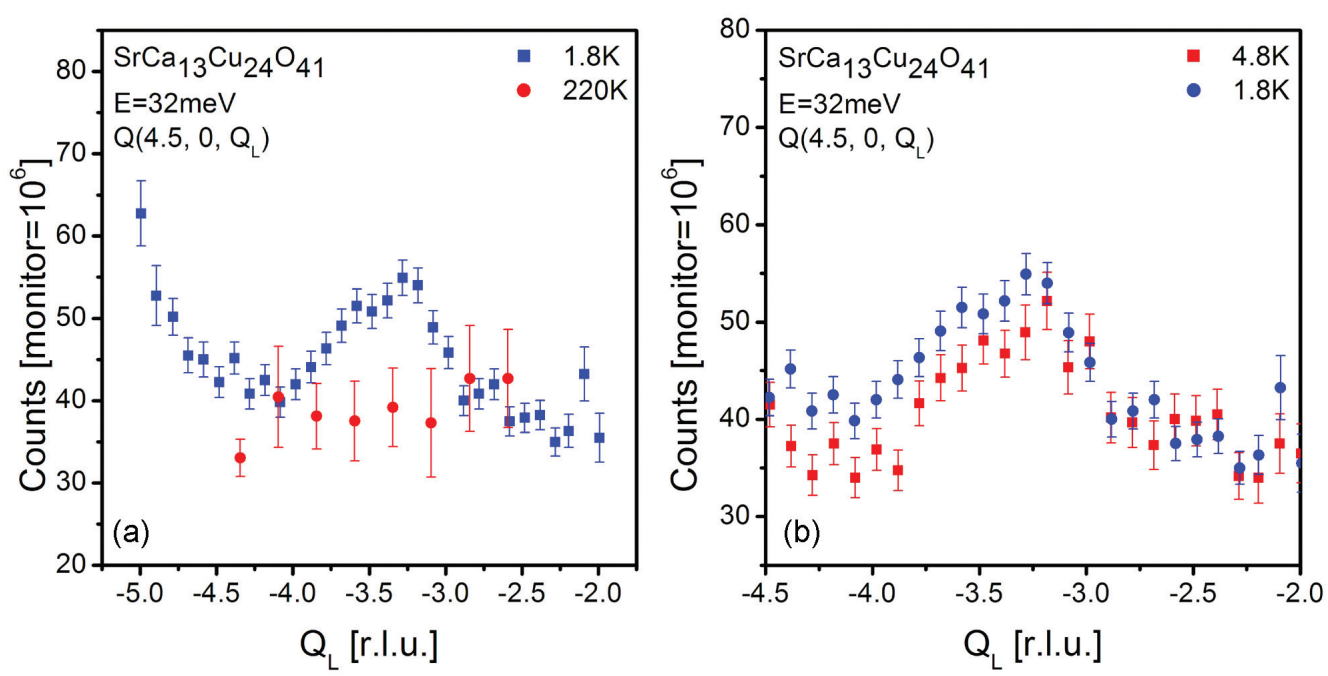

FIG. 7. (Color online) Constant energy ( $E=32 \mathrm{meV}$ ) scans along $Q_{L}$ direction: (a) $Q_{L}$ scans at $1.8 \mathrm{~K}$ and $220 \mathrm{~K}$; (b) $Q_{L}$ scans below and above $T_{N}$. The background scan at $220 \mathrm{~K}$ in (a) is scaled down to match the background at $1.8 \mathrm{~K}$ by a factor of 1.7 .

$\mathrm{Y}_{2} \mathrm{BaNiO}_{5}$ doped by $\mathrm{Mg}$ (Ref. 26). Ca doping in the same compound induced double peaked subgap excitation by the formation of AFM droplets induced by local FM bonds. ${ }^{27}$ Another example is the spin-Peierls gapped magnet $\mathrm{CuGeO}_{3}$ that undergoes a new AFM phase at low temperature when doping with nonmagnetic dopant Si (Ref. 14). These effects are fundamentally correlated to the staggered fields induced by the impurities in the system. ${ }^{28}$

Here we speculate that doped holes on the ladder sublattice form a nonmagnetic singlet state on $\mathrm{Cu}$ sites, just as the so-called Zhang-Rice singlet in other 2D network high temperature superconducting cuprates. ${ }^{29}$ Such a nonmagnetic singlet state plays a similar role to nonmagnetic impurities in driving $\mathrm{Cu}$ spins on the ladder sublattice into a long-range magnetic ordering state in $\mathrm{SrCa} 13$. Our speculation is supported by the theoretical work from Sandvik et al. ${ }^{28}$ They found that the nonmagnetic impurities on a two-leg spin-ladder lattice can induce staggered localized magnetic field near the impurity within a vicinity of $\sim 3.2$ lattice spacing, which can cause the long-range magnetic ordering of the spin-gapped lowdimensional magnetic system, such as spin-Peierls system and two-leg spin-ladder system. The magnetic structures proposed in this work agree well with the scenario discussed here. The FM clusters could be induced by the staggered magnetization and the size of the cluster matches well with the theoretical result. Furthermore, a modified t-J model proposed by J. Sabczynski et al $^{30}$ indicates that FM clusters are formed in the vicinity of doped holes on a 2D spin lattice of cuprates, explaining theoretically the ferromagnetic clusters found in our experiment. A more recent Monte-Carlo study confirmed the above conclusion, demonstrating that ferromagnetic clusters, which were called by the authors as "ferromagnetic bubbles", come into form when holes are introduced into an AFM matrix. ${ }^{31}$

When the spin ladder forms a long-range magnetic ordering, it is expected that the singlet ground state is weakened, and the spin excitation becomes gapless at certain wave vectors. Our recent work on the spin gap of the whole series of $\mathrm{Sr}_{14-x} \mathrm{Ca}_{x} \mathrm{Cu}_{24} \mathrm{O}_{41}$ have shown that the spin gap of the singlet-triplet excitation survived in all Ca-doped compounds with $x$ up to 13 (Ref. 11). As shown in Fig. 7(a), a clear spin-gap excitation peak was observed near $Q(4.5$, $0,3.5)$ in $\mathrm{SrCa} 13$ at $1.8 \mathrm{~K}$ but disappeared at $220 \mathrm{~K}$. We have also investigated the magnetic ordering impact on this singlet-triplet spin-gap excitation by measuring below $T_{N}$ $(1.8 \mathrm{~K})$ and above $T_{N}(4.8 \mathrm{~K})$. The result in Fig. $7(\mathrm{~b})$ shows that the excitation intensity was slightly enhanced at lower temperature. Therefore, these results unambiguously indicate the coexistence of the long-range magnetic ordering and the spin-singlet ground state in $\mathrm{SrCa} 13$, as previously suggested. ${ }^{24}$

A similar coexistence was also observed in several other low-dimensional magnetic systems with spin gap. For example, $\mathrm{CuGeO}_{3}$ is the well-known 1D spin-Peierls system. Nonmagnetic impurity (e.g., $\mathrm{Zn}$ or $\mathrm{Si}$ ) doping in this system causes the coexistence of long-range magnetic ordering and spin-Peierls (with spin gap) transitions in a wide doping range, which was confirmed via neutron scattering by J. P. Regnault et $a l .{ }^{14}$ Fukuyama et al. ${ }^{16}$ used a phase Hamiltonian to investigate the coexistence of the long-rang magnetic ordering and spin gap in this doped spin-Peierls system. They found that the lattice-order parameter and the spin-order parameter can coexist together in this system. Another example is $\mathrm{CsNiCl}_{3}$, which is the first $S=1$ Haldane chain system confirmed to have a spin gap. This magnet undergoes a long-range AFM phase transition at $\sim 4.8 \mathrm{~K}$. Below $T_{N}$, the Haldane gap still survives with enhanced intensity near the zone center $\left(\frac{1}{3}\right.$, $\left.\frac{1}{3}, 1\right)$. Another spin-wave mode from the $3 \mathrm{D}$ ordering phase shows up below $T_{N}$ (Ref. 32). Theoretical work attributes the long-range magnetic ordering to the interchain coupling at low temperature. ${ }^{17}$ The spin-gap excitation intensity of $\mathrm{SrCa} 13$ slightly increases below $T_{N}$, which is consistent with the observation in $\mathrm{CsNiCl}_{3}$ (Ref. 15) but different from Si-doped $\mathrm{CuGeO}_{3}$ (Ref. 14). Besides, we did not observe magnetic Bragg peak at the wave vector $(4.5,0,3.5)$, where the spin-gap excitation was observed. A detailed theoretical work may help us to understand the special characteristics of the coexisting ground state in this compound. 


\section{CONCLUSION}

In conclusion, a long-range magnetic ordering state was observed in the spin-ladder compound $\mathrm{SrCa} 13$ and confirmed by neutron diffraction. We found two possible magnetic structure models to explain the observed magnetic peak intensities by searching through a great amount of models. The magnetic models show that the magnetic ordering is due to the ordering of $\mathrm{Cu}$ moments along the $a$ axis on the spin-ladder sublattice. It was also found that the nearest $\mathrm{Cu}$ couplings along rungs and legs are mainly antiferromagnetic with some ferromagnetic clusters. The holes transferred from chains to ladders could be the main reason for this long-range magnetic transition.
In the magnetic ordered phase, the singlet-triplet excitation was confirmed by inelastic neutron scattering, evidencing the coexistence of the singlet ground state and long-range magnetic ordering in SrCa13.

\section{ACKNOWLEDGMENT}

Some of the authors (G. Deng, E. Pomjakushina, and K. Conder) gratefully acknowledge financial support from the Indo-Swiss Joint Research Program (ISJRP, Contract No. JRP122960) by the Swiss State Secretariat of Education and Research.
*Corresponding author: guochu.deng@ansto.gov.au

${ }^{1}$ F. D. M. Haldane, Phys. Lett. A 93, 464 (1983).

${ }^{2}$ E. Dagotto, J. Riera, and D. Scalapino, Phys. Rev. B 45, 5744 (1992).

${ }^{3}$ T. M. Rice, S. Gopalan, and M. Sigrist, Europhys. Lett. 23, 445 (1993).

${ }^{4}$ G. Y. Xu, J. F. DiTusa, T. Ito, K. Oka, H. Takagi, C. Broholm, and G. Aeppli, Phys. Rev. B 54, R6827 (1996).

${ }^{5}$ M. Azuma, Z. Hiroi, M. Takano, K. Ishida, and Y. Kitaoka, Phys. Rev. Lett. 73, 3463 (1994).

${ }^{6}$ M. Uehara, T. Nagata, J. Akimitsu, H. Takahashi, N. Mori, and K. Kinoshita, J. Phys. Soc. Jpn. 65, 2764 (1996).

${ }^{7}$ T. Vuletic, B. Korin-Hamzic, T. Ivek, S. Tomic, B. Gorshunov, M. Dressel, and J. Akimitsu, Phys. Rep. 428, 169 (2006).

${ }^{8}$ M. Matsuda and K. Katsumata, Phys. Rev. B 53, 12201 (1996).

${ }^{9}$ G. Deng, V. Pomjakushin, V. Petříček, E. Pomjakushina, M. Kenzelmann, and K. Conder, Phys. Rev. B 84, 144111 (2011).

${ }^{10}$ K.-i. Kumagai, S. Tsuji, M. Kato, and Y. Koike, Phys. Rev. Lett. 78, 1992 (1997).

${ }^{11}$ G. Deng, N. Tsyrulin, P. Bourges, D. Lamago, H. Ronnow, M. Kenzelmann, S. Danilkin, E. Pomjakushina, and K. Conder, Phys. Rev. B 88, 014504 (2013).

${ }^{12}$ M. Isobe, Y. Uchida, and E. Takayama-Muromachi, Phys. Rev. B 59, 8703 (1999).

${ }^{13}$ T. Nagata, H. Fujino, K. Satoh, N. Yamamori, J. Akimitsu, S. Katano, M. Nishi, K. Kakurai, M. Hiroi, M. Sera, N. Kobayashi, K. Tenya, H. Amitsuka, T. Takigawa, H. Inago, and T. Sakakibara, J. Phys. Soc. Jpn. 70, 2419 (2001).

${ }^{14}$ L. P. Regnault, J. P. Renard, G. Dhalenne, and A. Revcolevschi, Europhys. Lett. 32, 579 (1995).

${ }^{15}$ W. J. L. Buyers, R. M. Morra, R. L. Armstrong, M. J. Hogan, P. Gerlach, and K. Hirakawa, Phys. Rev. Lett. 56, 371 (1986).

${ }^{16}$ H. Fukuyama, T. Tanimoto, and M. Saito, J. Phys. Soc. Jpn. 65, 1182 (1996).
${ }^{17}$ S. Maslov and A. Zheludev, Phys. Rev. B 57, 68 (1998)

${ }^{18}$ G. Deng, D. M. Radheep, R. Thiyagarajan, E. Pomjakushina, S. Wang, N. Nikseresht, S. Arumugam, and K. Conder, J. Cryst. Growth 327, 182 (2011).

${ }^{19}$ J. Rodríguez-Carvajal, Physica B 192, 55 (1993).

${ }^{20}$ S. A. Carter, B. Batlogg, R. J. Cava, J. J. Krajewski, W. F. Peck, and T. M. Rice, Phys. Rev. Lett. 77, 1378 (1996).

${ }^{21}$ M. Azuma, Y. Fujishiro, M. Takano, M. Nohara, and H. Takagi, Phys. Rev. B 55, R8658 (1997).

${ }^{22}$ M. Kenzelmann, A. Zheludev, S. Raymond, E. Ressouche, T. Masuda, P. Böni, K. Kakurai, I. Tsukada, K. Uchinokura, and R. Coldea, Phys. Rev. B 64, 054422 (2001).

${ }^{23}$ D. E. Cox and V. J. Minkiewicz, Phys. Rev. B 4, 2209 (1971).

${ }^{24}$ T. Nagata, H. Fujino, J. Akimitsu, M. Nishi, K. Kakurai, S. Katano, M. Hiroi, M. Sera, and N. Kobayashi, J. Phys. Soc. Jpn. 68, 2206 (1999).

${ }^{25}$ S. Ohsugi, K. Magishi, S. Matsumoto, Y. Kitaoka, T. Nagata, and J. Akimitsu, Phys. Rev. Lett. 82, 4715 (1999).

${ }^{26}$ M. Kenzelmann, G. Xu, I. A. Zaliznyak, C. Broholm, J. F. DiTusa, G. Aeppli, T. Ito, K. Oka, and H. Takagi, Phys. Rev. Lett. 90, 087202 (2003).

${ }^{27}$ G. Y. Xu, G. Aeppli, M. E. Bisher, C. Broholm, J. F. DiTusa, C. D. Frost, T. Ito, K. Oka, R. L. Paul, H. Takagi, and M. M. J. Treacy, Science 289, 419 (2000).

${ }^{28}$ A. W. Sandvik, E. Dagotto, and D. J. Scalapino, Phys. Rev. B 56, 11701 (1997).

${ }^{29}$ F. C. Zhang and T. M. Rice, Phys. Rev. B 37, 3759 (1988).

${ }^{30}$ J. Sabczynski, M. Schreiber, and A. Sherman, Phys. Rev. B 48, 543 (1993).

${ }^{31}$ M. M. Maśka, M. Mierzejewski, E. A. Kochetov, L. Vidmar, J. Bonča, and O. P. Sushkov, Phys. Rev. B 85, 245113 (2012).

${ }^{32}$ R. M. Morra, W. J. L. Buyers, R. L. Armstrong, and K. Hirakawa, Phys. Rev. B 38, 543 (1988). 